\title{
Effects of palmitic acid on localization of embryo cell fate and blastocyst formation gene products
}

\author{
Michele D Calder(1) 1,2,3, Robert Chen ${ }^{2,3}$, Anastasia MacDonald ${ }^{2,3}$, Zoe MacNeily ${ }^{2,3}$, \\ Zuleika Chin Lai Leung ${ }^{1,2,3}$, Samira Adus ${ }^{2,3}$, Shiyu Cui ${ }^{2,3}$, Dean H Betts ${ }^{1,2,3}$, Basim Abu Rafea ${ }^{1,3}$ \\ and Andrew J Watson ${ }^{1,2,3}$ \\ ${ }^{1}$ Department of Obstetrics and Gynaecology, The University of Western Ontario, London, Ontario, Canada, \\ ${ }^{2}$ Department of Physiology and Pharmacology, The University of Western Ontario, London, Ontario, Canada and \\ ${ }^{3}$ The Children's Health Research Institute - Lawson Health Research Institute, London, Ontario, Canada
}

Correspondence should be addressed to M D Calder; Email: michele.calder@schulich.uwo.ca

\begin{abstract}
As obese and overweight patients commonly display hyperlipidemia and are increasingly accessing fertility clinics for their conception needs, our studies are directed at understanding the effects of hyperlipidemia on early pregnancy. We have focused on investigating palmitic acid (PA) and oleic acid (OA) treatment alone and in combination from the mouse two-cell stage embryos as a model for understanding their effects on the mammalian preimplantation embryo. We recently reported that PA exerts a negative effect on mouse two-cell progression to the blastocyst stage, whereas OA co-treatment reverses that negative effect. In the present study, we hypothesized that PA treatment of mouse embryos would disrupt proper localization of cell fate determining and blastocyst formation gene products and that co-treatment with OA would reverse these effects. Our results demonstrate that PA treatment significantly $(P<\mathbf{0 . 0 5})$ reduces blastocyst development and cell number but did not prevent nuclear localization of YAP in outer cells. PA treatment significantly reduced the number of $\mathrm{OCT}^{+}$and $\mathrm{CDX2}^{+}$nuclei. PA-treated embryos had lower expression of blastocyst formation proteins (E-cadherin, ZO-1 and Na/K-ATPase alpha1 subunit). Importantly, co-treatment of embryos with OA reversed PA-induced effects on blastocyst development and increased inner cell mass (ICM) and trophectoderm (TE) cell numbers and expression of blastocyst formation proteins. Our findings demonstrate that PA treatment does not impede cell fate gene localization but does disrupt proper blastocyst formation gene localization during mouse preimplantation development. OA treatment is protective and reverses PA's detrimental effects. The results advance our understanding of the impact of FFA exposure on mammalian preimplantation development.

Reproduction (2022) 163 133-143
\end{abstract}

\section{Introduction}

The World Health Organization defines obesity as a BMI of $30 \mathrm{~kg} / \mathrm{m}^{2}$ or greater (WHO 2000) and obesity is reaching epidemic proportions. The incidence of diabetes, hypertension, heart disease and high serum cholesterol demonstrates a graded increase as BMI rises (Paeratakul et al. 2002, Knight et al. 2010). Obese females face many challenges including menstrual irregularity, endometrial pathologies, higher miscarriage rates and infertility (Broughton \& Moley 2017). As BMI increases, women experience increased time-topregnancy and overall decreased fecundity (Wise et al. 2010). Furthermore, when obese women conceive, their pregnancies have increased incidence of preterm birth, high- and low-birth weight infants, increased risk of congenital anomalies (Knight et al. 2010, Schummers et al. 2015, Stang \& Huffman 2016), pre-eclampsia and gestational diabetes (Stang \& Huffman 2016). Assisted reproductive technologies (ARTs) are increasingly being required to achieve successful pregnancy in sub-fertile obese couples. Increasing BMI is positively correlated with pregnancy loss following ART treatment (Fedorcsák et al. 2004).

Obesity is accompanied by increased serum (Gaudet et al. 2012) and follicular fluid palmitic acid (PA) levels (Niu et al. 2014, Valckx et al. 2014). PA is a 16-carbon fully saturated fatty acid that increases the synthesis of harmful lipids and impedes normal organelle functioning (Yuzefovych et al. 2010, Palomer et al. 2018). It is the most abundantly saturated fatty acid in the human body (Carta et al. 2017). PA is present in many foods including meat, dairy and palm oil (Carta et al. 2017). The dysregulation of PA levels has detrimental longterm effects such as atherosclerosis, neurodegenerative disorders and cancer (Fatima et al. 2019).

Oleic acid $(\mathrm{OA})$ is an 18-carbon fatty acid with a single double bond (Lopez et al. 2014). It is the most abundant 
unsaturated fatty acid in human plasma (Palomer et al. 2018). OA can be obtained in the human diet through olive, canola and peanut oils (Lopez et al. 2014) and is a primary component of the Mediterranean diet which lessens disease incidence such as diabetes (MartınezGonzalez et al. 2008). OA decreases PA-induced apoptosis and ROS (Yuzefovych et al. 2010), decreases PA-induced inflammation and relieves ER stress (Palomer et al. 2018). We have recently reported that PA treatment reduces mouse blastocyst development in a concentration-dependent manner and alters ER stress pathway transcript levels, while co-treatment of OA with PA reversed these negative effects (Yousif et al. 2020). Interestingly, we observed a small percentage of PA (100 $\mu \mathrm{M})$ treated two-cell stage embryos that still cavitate, but for this study, we were especially interested in those that were unable to cavitate and become blastocysts.

Mammalian preimplantation development is characterized by a series of cleavage cell divisions from fertilized oocyte to multi-cellular blastocyst (Watson \& Barcroft 2001). The initial cleavages occur without concomitant embryo growth (Hardy et al. 1993), and eventually the cells compact and form a morula (Watson \& Barcroft 2001, Pfeffer 2018). At the mouse 32-cell stage, a cavity begins to form, leading to the blastocyst stage and the specification of two distinct cell lineages: the TE and ICM (Watson \& Barcroft 2001, Niwa et al. 2005).

OCT4 (POU5F1)is a pluripotency cell factor that gradually becomes restricted to the mouse ICM, acting to regulate transcription of target genes and maintain ICM pluripotency (Nichols et al. 1998). CDX2 is expressed in all cells at the 8-cell stage and gradually becomes restricted to the future trophectoderm in the blastocyst (Niwa et al. 2005). CDX2-deficient embryos have a poorly formed TE with deficiencies in maintaining tight junctions (Strumpf et al. 2005). OCT4 is downregulated when CDX2 reaches a specific threshold in mouse outer cells and collectively this asymmetry in cell lineage expression drives the formation of the ICM and TE (Niwa et al. 2005). CDX2 expression is induced by the transcription factor TEAD4 (Nishioka et al. 2009) and also requires Hippo pathway member YAP (Ota \& Sasaki 2008). Failure to properly generate the first cell lineages can prevent the initiation of pregnancy (Piliszek et al. 2016).

Blastocyst development depends on the timely expression of critical gene families including: E-cadherincatenin cell adhesion genes, the tight junction genes and $\mathrm{Na} / \mathrm{K}$-ATPase subunits (reviewed in Watson \& Barcroft 2001, Bell et al. 2008). Na/K-ATPase develops the ionic gradient which drives the movement of water through aquaporins across the epithelium to form the fluid-filled blastocyst cavity (Watson \& Barcroft 2001). The cell-tocell adhesion provided by E-cadherin-catenin and tight junctional proteins such as ZO-1 inhibits the leakage of blastocyst fluid and maintains a polarized $\mathrm{Na}^{+} / \mathrm{K}^{+}-$
ATPase distribution (Watson \& Barcroft 2001). These events are interconnected, as interrupting expression or activity of $\mathrm{Na} / \mathrm{K}$-ATPase in embryos affects localization of ZO-1 (Violette et al. 2006, Madan et al. 2007).

Because of the many fertility challenges obese women face and the importance of preimplantation development to successful pregnancy (Knight et al. 2010), we have pursued an understanding of how preimplantation development may be affected by PA and OA exposure. To date, research has not explored the effects of PA and OA on cell fate gene product and blastocyst formation gene product localization during preimplantation development. The primary objective was to determine how PA and OA treatment, alone and in combination, will affect YAP, OCT4, CDX2, $\mathrm{Na} / \mathrm{K}$ ATPase $\alpha-1$, E-cadherin and ZO-1 localization in mouse preimplantation embryos. We hypothesized that PA treatment of mouse preimplantation embryos would disrupt normal cell fate gene product and blastocyst formation gene product localization and that co-treatment with OA would reverse these effects. Our findings demonstrate that PA treatment does not impede cell fate gene product localization but reduces cell margin localization of blastocyst formation proteins. OA co-treatment reverses these effects of PA treatment. The results advance our understanding of the possible impact of obesity and FFA exposure on mammalian preimplantation development.

\section{Materials and methods}

\section{Animal source and ethics approval}

CD1 mice were acquired from Charles River Canada (SaintConstant, Quebec, Canada). The mice had free access to food and water while being housed in $12 \mathrm{~h}$ light: $12 \mathrm{~h}$ darkness cycles. All mice were handled according to the Canadian Council on Animal Care and Western University's Animal Care and Use Policies (protocol \#: 2018-075 to Dr Andrew J. Watson).

\section{Mouse superovulation and mating}

Four- to six-week-old female CD-1 mice received a 5-7.5 international units (IU) intraperitoneal (i.p.) injection with pregnant mare's serum gonadotropin (Folligon, Merck Animal Health, Canada). This was followed by a 5-7.5 IU i.p. injection of human chorionic gonadotrophin (Chorulon, Merck) 46-48 $\mathrm{h}$ later. Following the second injection, each female was placed in a cage with a single male CD-1 mouse (3-8 months of age) for mating overnight. The following morning, female mice were checked for presence of a seminal plug. Forty-six hours post-injection of hCG, female mice were sacrificed by $\mathrm{CO}_{2}$ asphyxiation (standard operating procedure). Their oviducts were collected and flushed with M2 flushing medium (Sigma-Aldrich). Flushed two-cell stage embryos were washed $3 \times$ in $50 \mu \mathrm{L}$ drops of potassium simplex optimization medium with amino acids (KSOMaa Evolve, Zenith Biotech, 
Canada) for OCT4/CDX2 and cell counting experiments. Due to discontinuation of this product, YAP, Na/K ATPase $\alpha$ 1, E-cadherin and ZO-1experiments were conducted with KSOM+AA (IVL04, Caisson Laboratories, Smithfield, UT). After washing, embryos were distributed equally among experimental treatment groups and cultured in $20 \mu \mathrm{L}$ drops under mineral oil (LiteOil LGOL-500, Cooper Surgical, Trumbull, CT) at a density of 1 embryo per $\mu \mathrm{L}$ for $46 \mathrm{~h}$ under a $5 \% \mathrm{CO}_{2}, 5 \% \mathrm{O}_{2}$ and $90 \% \mathrm{~N}_{2}$ culture atmosphere.

\section{Free-fatty acid (FFA) preparation and embryo culture}

A 20\% FFA-free BSA (A6003, Sigma) was prepared in PBS, filter sterilized and used to conjugate with PA (P0500, Sigma) or OA (75090, Sigma) as outlined in Alsabeeh et al. (2018). Stock PA and OA solutions were prepared by solubilizing each FFA in RNAse-free water and $\mathrm{NaOH}$ at $70^{\circ} \mathrm{C}$ to create a $20 \mathrm{mM}$ solution that was stored at $-20^{\circ} \mathrm{C}$. This stock was heated to $70^{\circ} \mathrm{C}$ and conjugated in a $1: 3(\mathrm{v} / \mathrm{v})$ ratio with $20 \%$ BSA initially, followed by a dilution to $500 \mu \mathrm{M}$ FFA solution in KSOM that was filtered and stored at $4{ }^{\circ} \mathrm{C}$. This results in a 2:1 molar ratio of PA or OA to BSA. The BSA control had a similar amount of added $\mathrm{NaOH}$. All treatment groups thus contained $0.6 \%$ added fatty acid-free BSA final concentration.

\section{$P A$ and $O A$ concentration response and treatment experiments}

$\mathrm{PA}$ and $\mathrm{OA}$ treatment concentrations were derived from concentration responsive experiments conducted and reported in our recent study (Yousif et al. 2020); $100 \mu \mathrm{M}$ PA had a negative effect on blastocyst development and 100 $\mu \mathrm{M}$ OA was able to overcome these deficits. PA is in the low physiological range, as 300-400 $\mu \mathrm{M}$ PA was measured in the regular chow-fed mouse oviduct (Yousif et al. 2020). Third trimester women with normal glucose tolerance had serum levels of $122 \mu \mathrm{M}$ PA and $83 \mu \mathrm{M}$ OA (Chen et al. 2010) which increased in obese women with gestational diabetes to 151 and $104 \mu \mathrm{M}$, respectively. For each experimental replicate, treatment groups consisted of 2- to 4-cell stage mouse embryos randomly allocated to the following treatments: (1) control KSOMaa medium $(120 \mu \mathrm{L}$ BSA control in a total volume of $300 \mu \mathrm{L}$ balance KSOM); (2) KSOMaa medium +100 $\mu \mathrm{M}$ PA $(60 \mu \mathrm{L}+60 \mu \mathrm{L}$ BSA control in $300 \mu \mathrm{L})$; 3) KSOMaa medium $+100 \mu \mathrm{M}$ OA $(60 \mu \mathrm{L}+60 \mu \mathrm{L}$ BSA control in $300 \mu \mathrm{L})$ and 4) KSOMaa + $100 \mu \mathrm{M} \mathrm{PA}(60 \mu \mathrm{L})$ and $100 \mu \mathrm{M} \mathrm{OA}(60 \mu \mathrm{L}$ in $300 \mu \mathrm{L})$. Embryos were cultured under a $5 \% \mathrm{CO}_{2}, 5 \% \mathrm{O}_{2}$ and $90 \% \mathrm{~N}_{2}$ atmosphere at $37^{\circ} \mathrm{C}$ for $46 \mathrm{~h}$ to assess progression to the blastocyst stage. Blastocysts were defined as embryos with a visible fluid-filled cavity of any size and the proportion was calculated based on number of two-cell cultured. Following this assessment, embryos were fixed for application of indirect immunofluorescence localization of OCT4, CDX2, YAP, $\mathrm{Na} / \mathrm{K}$-ATPase $\propto 1$ subunit, ZO-1 or E-cadherin combined with 4,6-diamindino-2-phenylindole (DAPI) nuclear staining, actin cytoskeleton staining with rhodamine phalloidin and confocal microscopy.

\section{Immunofluorescence and confocal microscopy}

Following culture, embryos in each treatment were fixed and permeabilized in 2\% paraformaldehyde in PBS for $30 \mathrm{~min}$. Embryos were stored in PIPES/HEPES/EGTA and $\mathrm{MgCl}_{2}$ (PHEM, Schliwa \& van Blerkom 1981) buffer at $4^{\circ} \mathrm{C}$ before staining. The preimplantation embryos were then blocked in 5\% normal donkey serum (Jackson ImmunoResearch) with $0.01 \%$ Triton $\mathrm{X}-100$ in PBS for at least $1 \mathrm{~h}$ at room temperature. Embryos were washed once in PBS. Then primary antibodies were added to antibody dilution buffer (ADB) consisting of PBS containing $0.5 \%$ normal donkey serum and $0.005 \%$ Triton- $X$ and incubated overnight at $4{ }^{\circ} \mathrm{C}$. Antibodies included (1) mouse anti-YAP (sc101199, Santa Cruz Biotechnology) diluted (1:100), (2) mouse anti-OCT3/4 (C-10, sc-5279, 1:50 Santa Cruz), (3) rabbit antiCDX2 (ab76541, 1:100; Abcam), (4) rabbit anti-E-cadherin (\#3195, Cell Signaling) or (5) rat anti-ZO1 (MABT11, EMD Millipore), diluted in ADB. Rabbit-sodium potassium ATPase alpha1 subunit antibody (ab237969, 1:100, Abcam) staining was performed completely in PHEM buffer (Betts et al. 1998). Donkey anti-mouse Alexa Fluor 488- (715-545-151, Jackson ImmunoResearch), donkey anti-rabbit fluorescein (711-095152, Jackson), donkey anti-rat fluorescein (712-095-153, Jackson) or donkey anti-rabbit Alexa Fluor 555-conjugated secondary antibodies (A31572, Thermo Fisher Scientific) were added and incubated at $4{ }^{\circ} \mathrm{C}$ overnight. Embryos were counterstained with final 1:1000 dilution of $1 \mathrm{mg} / \mathrm{mL}$ stock solution DAPI (D9542, Sigma) to stain all nuclei blue and 1:20 final dilution of $5 \mu \mathrm{g} / \mathrm{mL}$ stock solution of rhodamine phalloidin (P1951, Sigma) to stain the actin cytoskeleton red for $1 \mathrm{~h}$ at $37^{\circ} \mathrm{C}$. Processed embryos were mounted on glass bottom dishes in drops of KSOMaa and covered with mineral oil (Sigma) to prepare for confocal imaging. Confocal microscopy was performed using a Zeiss LSM800 laser scanning confocal microscope (Carl Zeiss Microscopy). Increments of $5 \mu \mathrm{m}$ were used to produce a Z-stack for each embryo. Laser strength settings were consistent between groups within a replicate. Z-stacks were saved as CZI files on Zen imaging program (Zeiss). Using the cell counter function, OCT4, CDX2, YAP and DAPI total nuclei were quantified manually in FIJI (open source softeare ImageJ available at https://imagej.net/software/ fiji/). Nuclei were counted as positive if they had fluorescence substantially above cytoplasmic levels, cells that were dividing had increased cytoplasmic staining for OCT4 and were not counted as positive.

\section{Quantitation of $\mathrm{Na}^{+} / \mathrm{K}^{+}$ATPase alpha1 subunit, E-cadherin and ZO1 fluorescence intensity}

$\mathrm{Na}^{+} / \mathrm{K}^{+}$ATPase alpha1 subunit, E-cadherin and ZO-1 fluorescence intensity were quantified using Zen and Ilastik (www.ilastik.org). From the embryo group Z-stacks czi files taken at $10 \times$, rectangular boxes were drawn around individual embryos and the entire stack of green channel was converted to a TIFF file. In Ilastik, a single bright embryo slice was chosen in each replicate experiment, and the foreground and background fluorescence were delineated. All the other TIFF files were then imported and calibrated against this standard. For each embryo, all tissue slices were summed from 16 to 
$185 \mu \mathrm{m}$ Z-stack slices to give an individual embryo value. The average fluorescence of the negative control embryos was subtracted, embryos that had lower fluorescence than the negative were set to 0 . For each antibody, there were at least two (OCT4 and CDX2), three (YAP, $\mathrm{Na}^{+} / \mathrm{K}^{+}$ATPase alpha1 subunit or E-cadherin) or four (ZO-1) different embryo collections subjected to separate staining and quantification protocols. There was a minimum of 22 to a maximum of 68 embryos evaluated per treatment.

\section{Statistical analysis}

GraphPad PRISM (https://www.graphpad.com/scientificsoftware/prism/) was employed to perform statistical analyses. For cell fate protein localization studies, each embryo examined represented a single $n$ value within a treatment. However, for assessments of developmental stage, a biological replicate consisted of a single pool of embryos within a treatment group. Blastocyst development was analyzed using a one-way ANOVA test, and means were compared to one another by an ad hoc Tukey's multiple comparisons test. Data analysis for DAPI-, OCT4-, CDX2- and YAP-positive nuclei included applying Kruskal-Wallis non-parametric ANOVA (as values were not normally distributed) with Dunn's multiple comparisons tests. Data analysis of $\mathrm{Na}+\mathrm{K}+$ ATPase, ZO-1 and E-cadherin fluorescence intensity was performed with non-parametric Kruskal-Wallis ANOVA with Dunn's multiple comparisons tests. For all tests, $P$ values of less than or equal to 0.05 were considered significantly different from one another.

\section{Results}

\section{PA alone reduces blastocyst formation and total cell number while development is restored in combination with OA development}

Preimplantation embryos from each treatment group were assessed using conventional light microscopy. Blastocysts were identified as having fluid-filled cavities (Fig. 1A) and were quantified as a percentage of all preimplantation embryos observed in each culture treatment group (Fig. 1C). A statistically significant decrease $(P<0.001)$ in the number of blastocysts was observed in the PA-treated group compared to all other groups (Fig. 1C), which is consistent with our previous study (Yousif et al. 2020). There were no significant differences in blastocyst developmental frequency between the control, $\mathrm{OA}$ alone and PA and $\mathrm{OA}$ combination (PA/OA) treatment groups (Fig. 1C).

We assessed the total number of DAPI-stained nuclei observed in each embryo composing each treatment group (Fig. 1B and D). PA-treated embryos displayed a significant reduction $(P<0.001)$ in total number of nuclei compared to all other treatment groups and control group (Fig. 1D). PA/OA had lower cell number than control $(P<0.04$, Fig. 1D). The majority of PA-treated embryos appeared as uncompacted cleavage
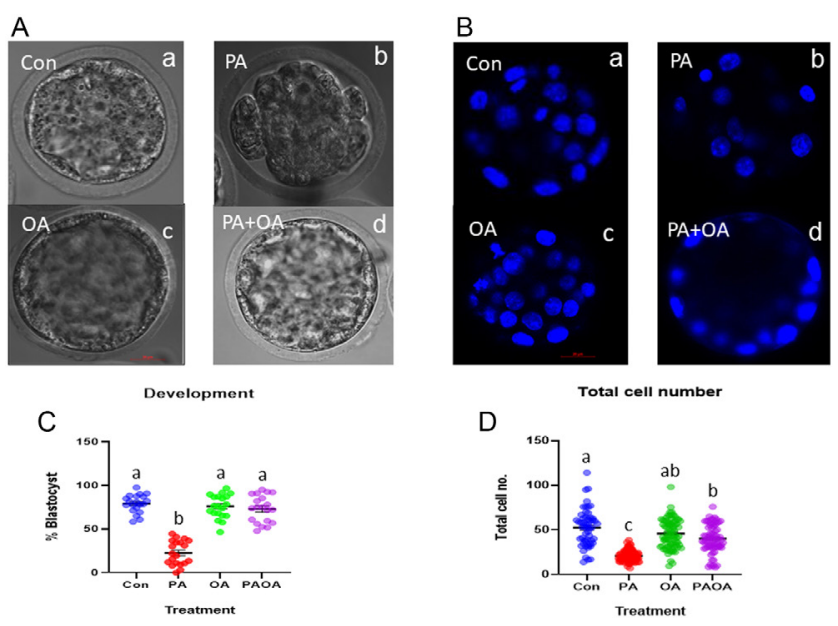

Figure 1 Representative phase contrast and DAPI-stained images from each treatment group. Brightfield (A) and confocal microscopy of preimplantation embryos imaged at $40 \times$ and stained with (B) DAPI (blue). (a) Control embryo, (b) PA-treated embryo, (c) OA-treated embryo and (d) PA and OA combination-treated embryo. Scale bars $=20 \mu \mathrm{m}$. (C) Data represent the mean percentage of blastocysts \pm S.E. $(n=20$, S.E.M.). (D) The mean cell numbers \pm S.E.M. observed for each treatment group. Groups that do not share the same letter are significantly different, $P<0.05$. (A, B, D) Control embryos $(n=5$ experimental replicates, 9-13 embryos per replicate), PA-treated embryos ( $n=5,11-36$ embryos per replicate), OA-treated embryos ( $n=5,13-21$ embryos per replicate) and PA and OA combinationtreated embryos ( $n=5,7-19$ embryos per replicate).

stage embryos (Fig. 2Ab). The mean cell number of the PA-treated group was approximately 21 cells.

\section{Effects of PA and OA treatment alone and in combination of YAP' nuclei}

The number of cells with YAP localized to the nucleus following each treatment was counted (Fig. 2A and C). The presence of binucleate cells in the PA-treated group was commonly observed (Fig. $2 \mathrm{Ab}$ and $\mathrm{Bb}$ ). Some $\mathrm{YAP}^{+}$ and $\mathrm{YAP}^{-}$cells were binucleate; however, cells with YAPnuclei were properly localized to the inside. PA-treated embryos displayed a significantly $(P<0.0001)$ lower number of $\mathrm{YAP}^{+}$nuclei compared to all other treatment groups (Fig. 2C). Interestingly, the proportion of $\mathrm{YAP}^{+}$ nuclei to total nuclei did not vary significantly $(P>0.05)$ between the control, PA and OA treatment groups (Fig. 2D). The proportion of $\mathrm{YAP}^{+}$nuclei to total cell nuclei in the PA and OA combination treatment was significantly $(P<0.05)$ higher than that observed for the control, OA and PA alone treatments (Fig. 2D).

\section{$P A$ reduced the number of $\mathrm{OCT}^{+}$nuclei, while $\mathrm{OA}$ in combination with PA restored $\mathrm{OCT}^{+}$number}

PA-treated embryos displayed a significantly lower $(P<0.0001)$ number of $\mathrm{OCT}^{+}$nuclei compared to the vehicle-treated control group and other treatment groups 


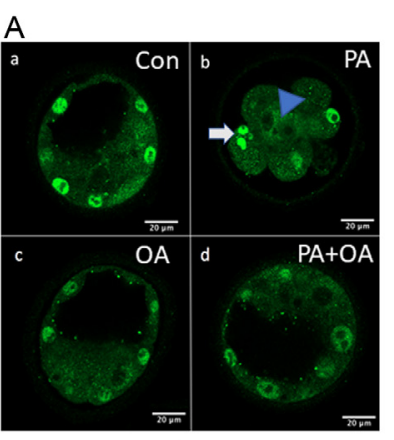

YAP

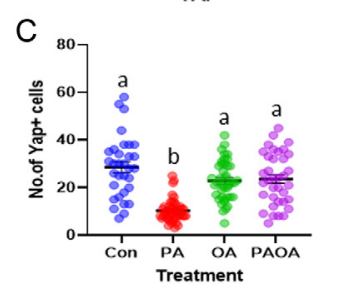

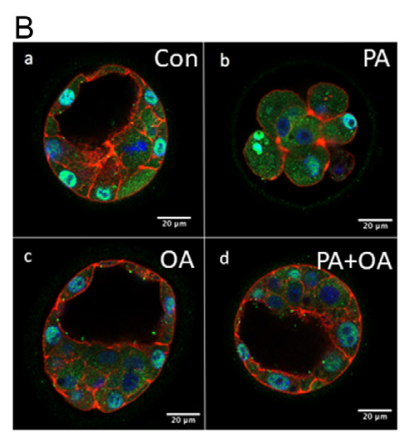

\%YAP

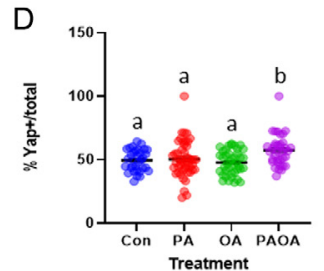

Figure 2 Representative YAP immunofluorescence images from each treatment group. OA attenuates the PA-induced decrease in number of cells expressing nuclear YAP. Representative confocal microscopy images of preimplantation embryos imaged at 40× following processing for indirect immunofluorescence (A) YAP (green), (B) rhodamine phalloidin (F-actin, red), DAPI (blue) representative merged images. (a) Control embryo, (b) PA-treated embryo, (c) OA-treated embryo and (d) PA and OA combination-treated embryo. Scale bars $=20 \mu \mathrm{m}$. PA-treated embryos frequently demonstrated binucleate cells. White arrow demonstrates binucleate cell with YAP ${ }^{+}$ nuclei, while blue arrowhead shows a cell with binucleate YAPnuclei. Control ( $n=3$ experimental replicates, 8-16 embryos per replicate), PA treated ( $n=3,11-19$ embryos per replicate), OA treated ( $n=3,13-15$ embryos per replicate) and PA and OA co-treated preimplantation embryos $(n=3,7-15$ embryos per replicate). (C) Data shown represent the value of the individual embryos and mean number of cells with nuclear YAP \pm S.E.M. in each embryo for each treatment group. (D) The value of the individual embryos and the mean proportion of nuclear YAP ${ }^{+}$cells \pm S.E.M. of total cells in all embryos in each treatment. Groups that do not share the same letters are significantly different, $P<0.05$.

(Fig. 3Ab and D). The mean proportion of $\mathrm{OCT}^{+}$nuclei in the control, OA and PA/OA embryos was significantly greater than the PA-treated embryos $(P<0.0001)$. The proportion of OCT4+ nuclei in OA-treated embryos $(P<0.005)$ and $P A$ and OA combination-treated embryos $(P<0.004)$ was lower than control embryos (Fig. 3E). There was no significant difference in the proportion of $\mathrm{OCT}_{4}^{+}$nuclei between OA- and PA/OA-treated embryos (Fig. 3E).

\section{$P A$ reduced the number of $C D X 2^{+}$nuclei, while $O A$ in combination with $P A$ restored $C D X 2+$ cell number}

The number of $\mathrm{CDX}^{+}$nuclei in the PA-treated embryos was significantly lower $(P<0.0001)$ compared to all other groups (Fig. 3Bb and F). The mean proportion of $\mathrm{CDX}^{+}$cells in PA-treated group was significantly lower $(P<0.0001)$ than the control and OA treatment groups and lower than the combined PA/OA group $(P<0.005$, Fig. 3G). Control, OA and PA/OA treatments were not significantly different from each other.

\section{PA reduced the amount of $\mathrm{Na}^{+} / \mathrm{K}^{+}$-ATPase $\alpha-1$ subunit, E-cadherin and ZO-1 membrane localization}

The level of $\mathrm{Na}^{+} / \mathrm{K}^{+}$-ATPase fluorescence intensity in PA-treated embryos was significantly lower $(P<0.005)$ compared to CON and $P<0.02$ for both OA and PA/OA (Fig. 4A, B and C). The level of E-cadherin fluorescence intensity was significantly lower $(P<0.02)$ in PA-treated embryos compared to the OA group (Fig. 5A, B and C). The level of ZO-1 fluorescence intensity was also significantly lower $(P<0.004)$ in PA-treated embryos compared to the CON group (Fig. 6A, B and C).

\section{Discussion}

Treatment of mouse embryos with PA resulted in impaired development similar to previous studies (Jungheim et al. 2011a, Yousif et al. 2020). The concentrations of PA and OA used are like those of serum levels in normal pregnant women of about $100 \mu \mathrm{M}$ (Chen et al. 2010), but PA can increase $30-60 \%$ in serum and follicular fluid in obese patients (Jungheim et al. 2011b, Valckx et al. 2014). Cleavage (O'Gorman et al. 2013) and pregnancy (Mirabi et al. 2017) in ART cycles are negatively correlated with follicular fluid PA concentrations. In the embryos treated with $100 \mu \mathrm{M}$ PA, the cell number averaged 21 cells, many embryos arrested at the 8-16 cell stage and $22 \%$ developed to the blastocyst stage. Short-term exposure of mammalian female gametes to PA showed long-lasting effects, as development was delayed (Desmet et al. 2020) and fetuses and liveborn animals were smaller following embryo transfer (Jungheim et al. 2011a). As well, gene expression was altered in both embryo and extra-embryonic tissue (Desmet et al. 2020). ER stress pathways are activated in mammalian female gametes and granulosa cells subjected to PA treatment in vitro (Wu et al. 2012, Yousif et al. 2020).

We observed that PA-treated embryos developed multi-nuclear cells. Multi-nuclear blastomeres in human embryos are quite common (Hardy et al. 1993), but they are rare in cultured CD1 mouse embryos. In human embryos, multi-nucleation is associated with aneuploidies (Staessen \& Van Steirteghem 1998). However, binucleation can resolve if it occurs at the two-cell stage (Aguilar et al. 2016, Paim \& FitzHarris 2020) but not later (Paim \& FitzHarris 2019). Paim and FitzHarris (2020) suggest that binucleate cells in later stage mouse embryos arise due to a failure of cytokinesis, as was observed earlier in arrested human embryos (Hardy et al. 1993). Zhang et al. (2010) demonstrated that PA treatment resulted in the formation of binuclear HeLa cells due to disrupted cytokinesis. Difficulties in completing cytokinesis may underlie the reduced 

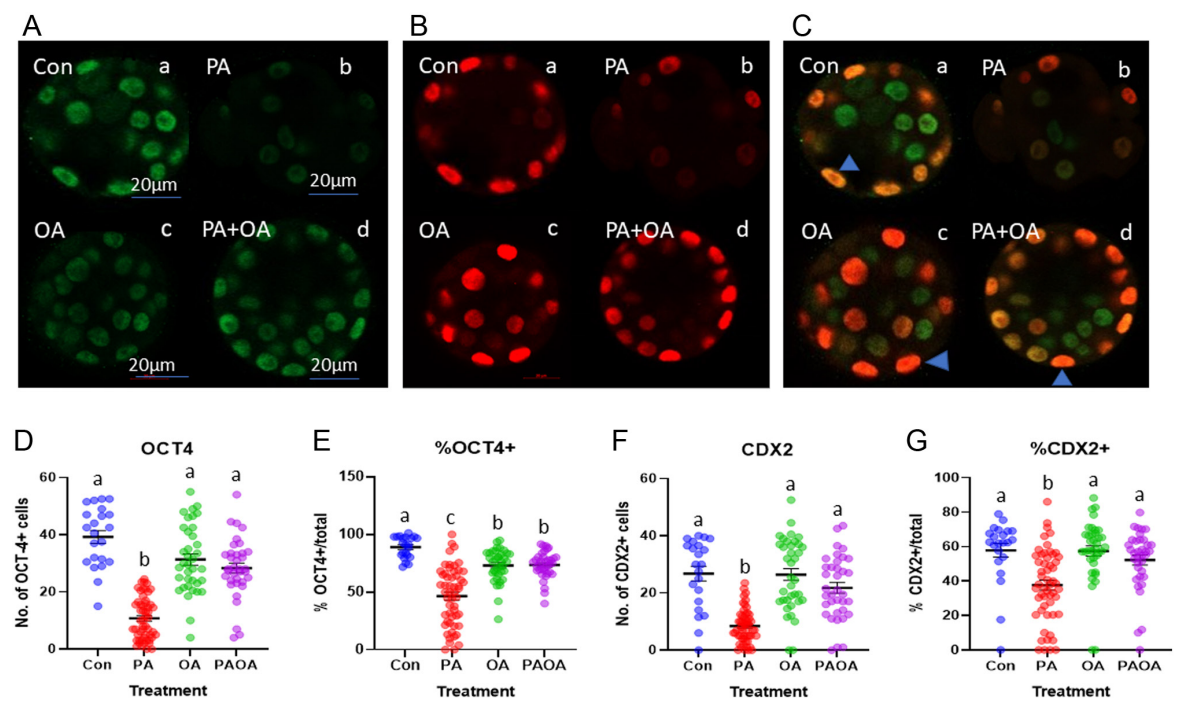

Figure 3 Representative OCT4 and CDX2 immunofluorescence images and quantification. Confocal microscopy images of preimplantation embryos imaged at 40× following processing for indirect immunofluorescence (A) OCT4 (green), (B) CDX2 (red), (C) merged representative OCT4 and CDX2 images. (a) Control embryo, (b) PA-treated embryo, (c) OA-treated embryo and (d) PA and OA combination-treated embryo. Scale bars $=20 \mu \mathrm{m}$. At early blastocyst stage, some outer nuclei still co-express OCT4 and CDX2 (yellow to orange staining), blue arrowheads. (D) Data shown represent the value of the individual embryos and the mean total OCT4 $4^{+}$nuclei \pm S.E.M. for each treatment group. (E) The mean proportion of OCT4 ${ }^{+}$nuclei \pm S.E.M. of total cells in each treatment. (F) Data shown represent the mean total CDX2+ nuclei \pm S.E.M. for each treatment group. (G) The mean proportion of $\mathrm{CDX}^{+}$nuclei \pm S.E.M. of total cells in each treatment. Groups that do not share the same letters are significantly different, $P<0.05$. Control embryos $(n=2$ experimental replicates, $10-12$ embryos per replicate), PA-treated embryos $(n=2$, 18-36 embryos per replicate), OA-treated embryos $(n=2,17-20$ embryos per replicate) and PA and OA combination-treated embryos $(n=2$, 17-19 embryos per replicate).
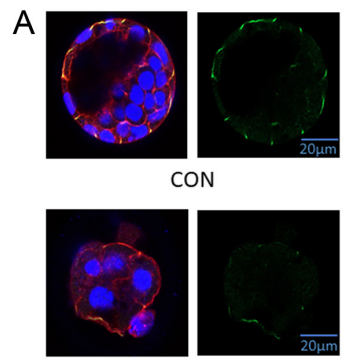

PA

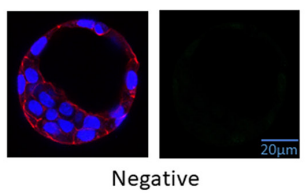

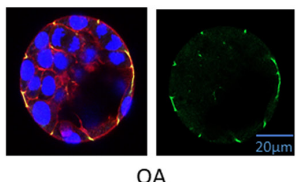

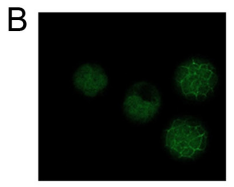

CON

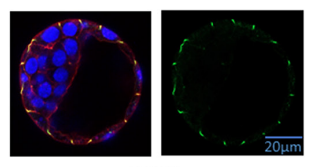

PAOA

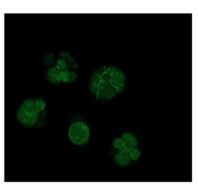

PA

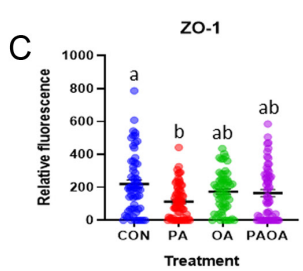

Treatment

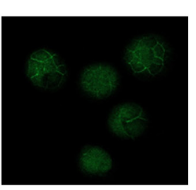

OA

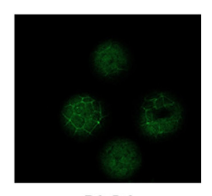

PAOA

Figure 4 Representative $\mathrm{Na}^{+} / \mathrm{K}^{+}$ATPase $\alpha-1$ immunofluorescence images from each treatment group. Confocal microscopy images of preimplantation embryos imaged at $40 \times$ following processing for indirect immunofluorescence (A) $\mathrm{Na}^{+} / \mathrm{K}^{+}$ATPase $\alpha-1$ subunit (green), F-actin (red), DAPI (blue), merged images on left panel and $\mathrm{Na}^{+} / \mathrm{K}^{+}$ATPase $\alpha-1$ subunit alone on right panel. (a) Control embryo, (b) PA-treated embryo, (c) OA-treated embryo and (d) PA and OA combination-treated embryo. Scale bars $=20 \mu \mathrm{m}$. (B) Embryo group photo of $\mathrm{Na}^{+} / \mathrm{K}^{+} \mathrm{ATPase} \alpha-1$ (green), $10 \times$ magnification, (C) quantification of $\mathrm{Na}^{+} / \mathrm{K}^{+}$ATPase $\alpha-1$ intensity in each treatment group. Data shown represent the values of the individual embryos and the mean $\mathrm{Na}^{+} / \mathrm{K}^{+}$ATPase $\alpha$ - 1 fluorescence \pm S.E.M. for each treatment group. Bars without letters in common are significantly different, $P<0.05$. Control embryos $(n=3$ experimental replicates, $13-16$ embryos per replicate), PA-treated embryos $(n=3$, 12-21 embryos per replicate), OA-treated embryos $(n=3,12-15$ embryos per replicate) and PA and OA combination-treated embryos $(n=3$, 14-15 embryos per replicate). 


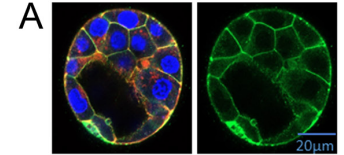

CON

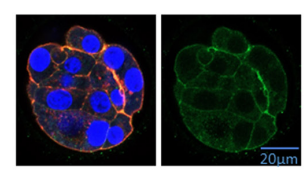

PA

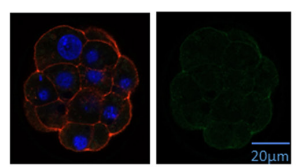

Negative

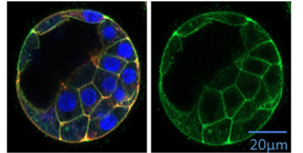

$\mathrm{OA}$

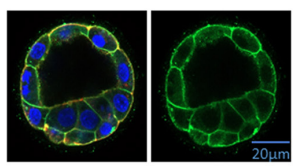

PAOA

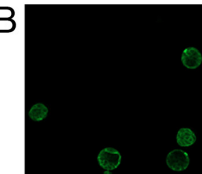

CON

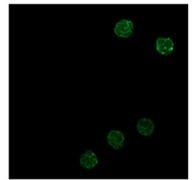

PA

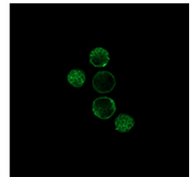

$\mathrm{OA}$

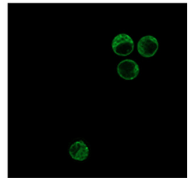

PAOA

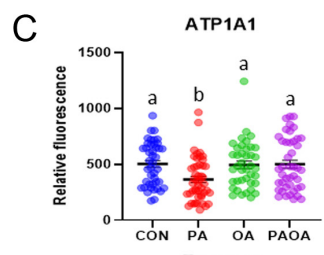

Figure 5 Representative E-cadherin immunofluorescence images from each treatment group. Confocal microscopy images of preimplantation embryos imaged at $40 \times$ following processing for indirect immunofluorescence (A) E-cadherin (green), F-actin (red), DAPI (blue), merged images on left panel and E-cadherin alone on right panel. (a) Control embryo, (b) PA-treated embryo, (c) OA-treated embryo and (d) PA and OA combination-treated embryo. Scale bars $=20 \mu \mathrm{m}$. (B) Embryo group photo of E-cadherin (green), 10× magnification (a) Control embryo, (b) PA-treated embryo, (c) OA-treated embryo and (d) PA and OA combination-treated embryo. (C) Quantification of E-cadherin intensity in each treatment group. Data shown represent the values of the individual embryos and the mean E-cadherin fluorescence \pm S.E.M. for each treatment group. Bars without letters in common are significantly different, $P<0.05$. Control embryos $(n=3$ experimental replicates, $15-20$ embryos per replicate), PA-treated embryos ( $n=3,17-19$ embryos per replicate), OA-treated embryos ( $n=3,12-20$ embryos per replicate) and PA and OA combination-treated embryos ( $n=3,14-18$ embryos per replicate).
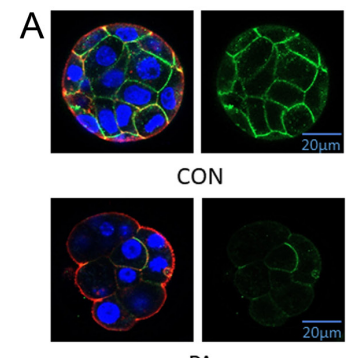

CON

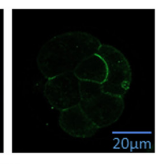

PA

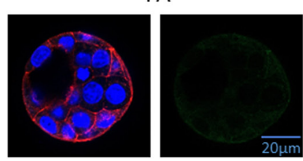

Negative
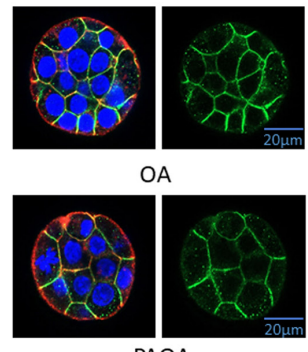

PAOA

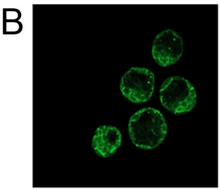

CON

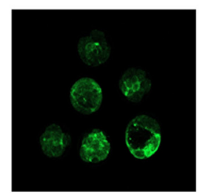

PA

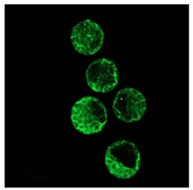

$\mathrm{OA}$

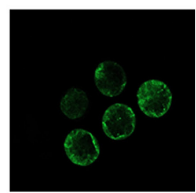

PAOA

C

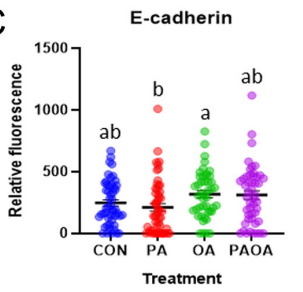

Figure 6 Representative ZO-1 images from each treatment group. Confocal microscopy images of preimplantation embryos imaged at 40x following processing for indirect immunofluorescence (A) ZO-1 subunit (green), F-actin (red), DAPI (blue), merged images on left panel, ZO-1 alone on right panel. (a) Control embryo, (b) PA-treated embryo, (c) OA-treated embryo and (d) PA and OA combination-treated embryo. Scale bars $=20 \mu \mathrm{m}$. (B) Embryo group photo of ZO-1 (green), 10× magnification. (C) Quantification of ZO-1 intensity in each treatment group. Data shown represent the values of the individual embryos and the mean ZO-1 fluorescence \pm S.E.M. for each treatment group. Bars without letters in common are significantly different, $P<0.05$. Control embryos ( $n=4$ experimental replicates, $11-18$ embryos per replicate), PA-treated embryos ( $n=4,10-24$ embryos per replicate), OA-treated embryos $(n=4,13-16$ embryos per replicate) and PA and OA combination-treated embryos $(n=4,13-16$ embryos per replicate). 
cell numbers of the PA treatment group in the present study. Further studies are required to determine whether this is the case and when binucleation is occurring in PA-treated 2-cell embryos.

While we have an excellent understanding of the basic mechanisms and molecular players involved in cell lineage specification in mammalian embryos, there is more to learn about governing mechanisms that coordinate the timing of cell fate events and their sequence (Piliszek et al. 2016). Early blastomeres transition from totipotency to obtaining positional information (outside or inside cells), triggering polarization, adherens junction cell to cell adhesion, and compaction leading to epithelialization and differentiation of TE cells (Piliszek et al. 2016). The role of the Hippo pathway in TE differentiation is to differentially regulate YAP phosphorylation in TE and ICM development (Nishioka et al. 2009, Anani et al. 2014, Sasaki 2017). Normally following compaction at the 8-cell stage, each blastomere acquires polarity (Johnson et al. 1986). At the 16-cell stage, nuclear YAP is expressed in the outer cells, controlled mainly by blastomeres inheriting apical membrane components (Anani et al. 2014, Hirate et al. 2015, Sasaki 2017). In the outer cells, i.e. TE progenitors, Hippo activity is inactivated thus allowing YAP to localize to the nucleus and interact with TEA domain transcription factor (TEAD) resulting in CDX2 expression and TE cell differentiation (Nishioka et al. 2009, Anani et al. 2014, Sasaki 2017). In contrast, the Hippo pathway is active in the ICM which results in phosphorylated YAP that remains cytoplasmic (Nishioka et al. 2009, Anani et al. 2014, Sasaki 2017). By the 32-cell stage, differential activation of Hippo signaling in the two-cell lineages is cemented by the formation of stable E-cadherin-mediated adherens junctions (Nishioka et al. 2009, Anani et al. 2014, Sasaki 2017). PA-treated embryos were predominantly 8-16-cells; still, YAP immunofluorescence localization appeared to be correct for regulating cell fate in outer cells, i.e. nuclear in outer cells. The proper localization of YAP may indicate that PA treatment did not alter membrane structure or domain inheritance. Since they are chronological at a normal developmental time point for these mechanisms to reveal themselves, we suggest that the PA-treated embryos were simply activating a normal cell fate developmental program according to the polar or apolar status of the blastomeres to the best of their ability.

The number of $\mathrm{YAP}^{+}$nuclei was lower in the PA group in comparison to other groups; however, the proportion of YAP+ nuclei was unaffected. PA embryos including cleavage stage embryos displayed nuclear YAP immunofluorescence. Other studies have found that PA reduced the number of endothelial cells with nuclear YAP (Yuan et al. 2017); however, studies with $\beta$-cell lines have shown that PA induced nuclear localization of YAP (Deng et al. 2016). Inhibition of YAP resulted in increased PA-induced apoptosis, suggesting that nuclear YAP may have a protective role in $\beta$-cells (Deng et al. 2016). Since, the proportion of $\mathrm{YAP}^{+}$nuclei did not vary significantly between PA and control groups, we would propose that cell lineage specification mechanisms were activated, but for the majority of PA-treated embryos, this was not sufficient to enable cavitation and blastocyst formation.

We observed lower numbers and proportion of $\mathrm{OCT}_{4}{ }^{+}$cells in PA-treated embryos, which is indicative of ICM. Similarly, the proportion of ICM cells was lower in embryos retrieved from high-fat diet fed mice (Minge et al. 2008, McPherson et al. 2015) and the number of ICM cells was lower in embryos from overweight women (Leary et al. 2015). CDX2 ${ }^{+}$cell numbers and proportion were also reduced in PA-treated embryos, which is indicative of reduced numbers of TE cells. Similarly, the number ofTE cells is reduced in embryos from overweight women (Leary et al. 2015). Both decreased proliferation and increased apoptosis were observed in trophoblast stem cells cultured with PA (Jungheim et al. 2011a). The reduction in total cell number as well as both ICM and TE cells suggests that PA treatment promotes metabolic changes within the early embryo (McKeegan \& Sturmey 2011). Leary et al. (2015) observed reductions in glucose uptake, alterations in amino acid turnover and increases triglyceride storage in embryos from overweight women.

We also observed that PA treatment reduced the fluorescent levels of $\mathrm{Na}^{+} / \mathrm{K}^{+}$ATPase, E-cadherin and ZO1 properly localized to blastomere cell margins. Exposure to high-fat diet reduced mRNA expression and reduced assembly of tight junctions and adherens junctions as well as $\mathrm{Na}^{+} / \mathrm{K}^{+}$ATPase expression and function in corneal cells (Bu et al. 2020). In addition, increased amounts of PA in the lipid bilayer reduced $\mathrm{Na}^{+} / \mathrm{K}^{+}$ATPase function (Gianfrancesco et al. 2019). $\mathrm{Na}^{+} / \mathrm{K}^{+}$ATPase activity is linked to membrane fluidity and negatively associated with levels of saturated fatty acids in erythrocytes (Rodrigo et al. 2014). Similarly, palmitate decreased integrity of tight and adherens junctions and increased permeability of the gut epithelium (Ghezzal et al. 2020). Failure to establish these junctions appropriately and produce $\mathrm{Na} / \mathrm{K}$-ATPase activity required for establishing a trans-trophectoderm ion gradient is likely the main factor underlying the reduced ability of PA-treated embryos to cavitate and form blastocysts (Barcroft et al. 1998, Betts et al. 1998, Watson \& Barcroft 2001). Furthermore, blastocyst formation gene and cell fate promoting gene expression are interconnected, as CDX2-deficient embryos have deficiencies in forming and regulating trophectoderm apical tight junctions (Strumpf et al. 2005) and ZO-1 gene knockout decreased expression of OCT4 and CDX2 (Wang et al. 2008).

The total cell number in blastocysts and the proportion of ICM cells are important determinants 
of embryo viability following transfer (Papaionnou \& Ebert 1995, Lane \& Gardner 1997). However, a precise cell number is not required for blastocyst formation to occur. For example, it is possible to split embryos in half and still observe cavitation in embryos with half their normal cell number (Tarkowski 1959, Papaionnou \& Ebert 1995). Since early mammalian embryos have this capacity to cavitate with significantly reduced cell number, we were compelled to determine if their reduced ability to cavitate was associated with an alteration in the expression of known blastocyst formation genes including E-cadherin, ZO- 1 and $\mathrm{Na} / \mathrm{K}-$ ATPase alpha 1 subunit genes. We have extensive experience with these gene products and have significantly helped with the understanding of their essential roles in driving cavitation and thus blastocyst formation (Barcroft et al. 1998, Betts et al. 1998, Watson \& Barcroft 2001, Violette et al. 2006). The fluorescence signal of all three blastocyst formation genes was significantly reduced in 48-h PA-treated embryos. We thus conclude that overall, it is likely that PA treatment impedes proper blastocyst formation gene product expression, and while cell fate gene products are properly localized in PA-treated embryos, their reduced ability to cavitate could be due to a disruption in normal E-cadherin, ZO-1 and $\mathrm{Na} / \mathrm{K}$-ATPase alpha subunit expression.

In conclusion, our study has reinforced the harmful effects of PA exposure on mouse embryo preimplantation development. Our observations are applicable of understanding the consequences that obesity may impose on human preimplantation embryo development as FFA within follicular fluid is positively correlated with BMI (Valckx et al. 2014) and negatively correlated with oocyte quality (Jungheim et al. 2011b). PA and other saturated fatty acids in the Western diet may hinder the growth of the embryo and lead to lower conception rates. In this study, treatment of twocell preimplantation mouse embryos with $100 \mu \mathrm{M}$ PA significantly reduced total embryo cell number and the numbers of $\mathrm{YAP}^{+}, \mathrm{OCT}^{+}$, and $\mathrm{CDX} 2^{+}$expressing nuclei as well as decreasing cell adhesion, tight junction and $\mathrm{Na}^{+} / \mathrm{K}^{+}$ATPase proteins in the blastomere membranes. In contrast, OA co-treatment alleviates these effects of PA treatment. There are many other FFAs that should be investigated for their influence on preimplantation development, and as progress continues in this manner, eventual understanding of the full possible effects of FFA exposure to early development will unfold. It will be important to conduct further experiments that are directed at dissecting stage-specific and treatment time course variation effects of PA and OA treatment on preimplantation development. Our findings offer potential therapeutic interventions as supplementation of embryo culture medium with OA may improve development in obese patients with elevated PA serum levels.

\section{Declaration of interest}

The authors declare that there is no conflict of interest that could be perceived as prejudicing the impartiality of the research reported.

\section{Funding}

This work was supported by a grant from the Canadian Institutes of Health Research (CIHR) to D H B, B A R and $\mathrm{A} J \mathrm{~W}$.

\section{Author contribution statement}

R C, A M, Z C L L, S A, S C, Z M and M D C performed acquisition of data, analysis and interpretation; M D C, A J W assisted with experimental conception and design; $M D C$, R C, A J W and D H B drafted the manuscript; M D C, A J W, D H B and B A R final approval of article.

\section{References}

Aguilar J, Rubio I, Muñoz E, Pellicer A \& Meseguer M 2016 Study of nucleation status in the second cell cycle of human embryo and its impact on implantation rate. Fertility and Sterility 106 291.e2-299.e2. (https://doi.org/10.1016/j.fertnstert.2016.03.036)

Alsabeeh N, Chausse B, Kakimoto PA, Kowaltowski AJ \& Shirihai O 2018 Cell culture models of fatty acid overload: problems and solutions. Biochimica et Biophysica Acta: Molecular and Cell Biology of Lipids 1863 143-151. (https://doi.org/10.1016/j.bbalip.2017.11.006)

Anani S, Bhat S, Honma-Yamanaka N, Krawchuk D \& Yamanaka Y 2014 Initiation of Hippo signaling is linked to polarity rather than to cell position in the pre-implantation mouse embryo. Development 141 2813-2824. (https://doi.org/10.1242/dev.107276)

Barcroft LC, Hay-Schmidt A, Caveney A, Gilfoyle E, Overstrom EW, Hyttel P \& Watson AJ 1998 Trophectoderm differentiation in the bovine embryo: characterization of a polarized epithelium. Journal of Reproduction and Fertility 114 327-339. (https://doi.org/10.1530/ jrf.0.1140327)

Bell CE, Calder MD \& Watson AJ 2008 Genomic RNA profiling and the programme controlling preimplantation mammalian development. Molecular Human Reproduction 14 691-701. (https://doi.org/10.1093/ molehr/gan063)

Betts DH, Barcroft LC \& Watson AJ $1998 \mathrm{Na} / \mathrm{K}-\mathrm{ATPase}-$ mediated ${ }^{86} \mathrm{Rb}^{+}$ uptake and asymmetrical trophectoderm localization of $\alpha 1$ and $\alpha 3$ $\mathrm{Na} / \mathrm{K}-\mathrm{ATPa} e$ isoforms during bovine pre-attachment development. Developmental Biology $197 \quad 77-92 . \quad$ (https://doi.org/10.1006/ dbio.1998.8874)

Broughton DE \& Moley KH 2017 Obesity and female infertility: potential mediators of obesity's impact. Fertility and Sterility 107 840-847. (https:// doi.org/10.1016/j.fertnstert.2017.01.017)

Bu J, Yu J, Wu Y, Cai X, Li K, Tang L, Jiang N, Jeyalatha MV, Zhang M, Sun H et al. 2020 Hyperlipidemia affects tight junctions and pump function in the corneal endothelium. American Journal of Pathology 190 563-576. (https://doi.org/10.1016/j.ajpath.2019.11.008)

Carta G, Murru E, Banni S \& Manca C 2017 Palmitic acid: physiological role, metabolism and nutritional implications. Frontiers in Physiology $\mathbf{8}$ 902. (https://doi.org/10.3389/fphys.2017.00902)

Chen X, Schol TO, Leskiw M, Savaille J \& Stein TP 2010 Differences in maternal circulating fatty acid composition and dietary fat intake in women with gestational diabetes mellitus or mild gestational hyperglycemia. Diabetes Care 33 2049-2054. (https://doi.org/10.2337/ dc10-0693)

Deng Y, Matsui Y, Pan W, Li Q \& Lai ZC 2016 Yap1 plays a protective role in suppressing free fatty acid-induced apoptosis and promoting beta-cell survival. Protein and Cell 7 362-372. (https://doi.org/10.1007/s13238016-0258-5) 
Desmet KLJ, Marei WFA, Richard C, Sprangers K, Beemster GTS, Meysman P, Laukens K, Declerck K, Vanden Berghe W, Bols PEJ et al. 2020 Oocyte maturation under lipotoxic conditions induces carryover transcriptomic and functional alterations during post-hatching development of good-quality blastocysts: novel insights from a bovine embryo-transfer model. Human Reproduction 35 293-307. (https://doi. org/10.1093/humrep/dez248)

Fatima S, Hu X, Gong RH, Huang C, Chen M, Wong HLX, Bian Z \& Kwan HY 2019 Palmitic acid is an intracellular signaling molecule involved in disease development. Cellular and Molecular Life Sciences 76 2547-2557. (https://doi.org/10.1007/s00018-019-03092-7)

Fedorcsák P, Dale PO, Storeng R, Ertzeid G, Bjercke S, Oldereid N, Omland AK, Abyholm T \& Tanbo T 2004 Impact of overweight and underweight on assisted reproduction treatment. Human Reproduction 19 2523-2528. (https://doi.org/10.1093/humrep/deh485)

Gaudet MM, Falk RT, Stevens RD, Gunter MJ, Bain JR, Pfeiffer RM, Potischman N, Lissowska J, Peplonska B, Brinton LA et al. 2012 Analysis of serum metabolic profiles in women with endometrial cancer and controls in a population-based case-control study. Journal of Clinical Endocrinology and Metabolism 97 3216-3223. (https://doi.org/10.1210/ jc.2012-1490)

Ghezzal S, Postal BG, Quevrain E, Brot L, Seksik P, Leturque A, Thenet S \& Carriere V 2020 Palmitic acid damages gut epithelium integrity and initiates inflammatory cytokine production. Biochimica et Biophysica Acta: Molecular and Cell Biology of Lipids 1865 158530. (https://doi. org/10.1016/j.bbalip.2019.158530)

Gianfrancesco MA, Dehairs J, L'homme L, Herinckx G, Esser N, Jansen O, Habraken Y, Lassence C, Swinnen JV, Rider MH et al. 2019 Saturated fatty acids induce NLRP3 activation in human macrophages through $\mathrm{K}^{+}$efflux resulting from phospholipid saturation and $\mathrm{Na}$, K-ATPase disruption. Biochimica et Biophysica Acta: Molecular and Cell Biology of Lipids 1864 1017-1030. (https://doi.org/10.1016/j. bbalip.2019.04.001)

Hardy K, Winston RM \& Handyside AH 1993 Binucleate blastomeres in preimplantation human embryos in vitro: failure of cytokinesis during early cleavage. Journal of Reproduction and Fertility 98 549-558. (https://doi.org/10.1530/jrf.0.0980549)

Hirate Y, Hirahara S, Inoue K, Kiyonari H, Niwa H \& Sasaki H 2015 ParaPKC-dependent and -independent mechanisms cooperatively control cell polarity, Hippo signaling, and cell positioning in 16-cell stage mouse embryos. Development, Growth and Differentiation 57 544-556. (https://doi.org/10.1111/dgd.12235)

Johnson MH, Maro B \& Takeichi M 1986 The role of cell adhesion in the synchronization and orientation of polarization in 8-cell mouse blastomeres. Journal of Embryology and Experimental Morphology 93 239-255. (https://doi.org/10.1242/dev.93.1.239)

Jungheim ES, Louden ED, Chi MM, Frolova AI, Riley JK \& Moley KH 2011 a Preimplantation exposure of mouse embryos to palmitic acid results in fetal growth restriction followed by catch-up growth in the offspring. Biology of Reproduction 85 678-683. (https://doi.org/10.1095/ biolreprod.111.092148)

Jungheim ES, Macones GA, Odem RR, Patterson BW, Lanzendorf SE, Ratts VS \& Moley KH $2011 b$ Associations between free fatty acids, cumulus oocyte complex morphology and ovarian function during in vitro fertilization. Fertility and Sterility 95 1970-1974. (https://doi. org/10.1016/j.fertnstert.2011.01.154)

Knight M, Kurinczuk J, Spark P, Brocklehurst P \& UK Obstetric Surveillance System 2010 Extreme obesity in pregnancy in the United Kingdom. Obstetrics and Gynecology 115 989-997. (https://doi. org/10.1097/AOG.0b013e3181da8f09)

Lane M \& Gardner DK 1997 Differential regulation of mouse embryo development and viability by amino acids. Journal of Reproduction and Fertility 109 153-164. (https://doi.org/10.1530/jrf.0.1090153)

Leary C, Leese HJ \& Sturmey RG 2015 Human embryos from overweight and obese women display phenotypic and metabolic abnormalities. Human Reproduction 30 122-132. (https://doi.org/10.1093/humrep/ deu276)

Lopez S, Bermudez B, Montserrat-de la Paz S, Jaramillo S, Varela LM, Ortega-Gomez A, Abia R \& Muriana FJ 2014 Membrane composition and dynamics: a target of bioactive virgin olive oil constituents. Biochimica et Biophysica Acta 1838 1638-1656. (https://doi. org/10.1016/j.bbamem.2014.01.007)
Madan P, Rose K \& Watson AJ 2007 Na/K-ATPase $\beta 1$ subunit expression is required for blastocyst formation and normal assembly of trophectoderm tight junction-associated proteins. Journal of Biological Chemistry 282 12127-12134. (https://doi.org/10.1074/jbc.M700696200)

Martınez-Gonzalez MA, de la Fuente-Arrillaga C, Nunez-Cordoba JM, Basterra-Gortari FJ, Beunza JJ, Vazquez Z, Benito S, Tortosa A \& Bes-Rastrollo M 2008 Adherence to Mediterranean diet and risk of developing diabetes: prospective cohort study. BMJ 336 1348-1351. (https://doi.org/10.1136/bmj.39561.501007.BE)

McKeegan PJ \& Sturmey RG 2011 The role of fatty acids in oocyte and early embryo development. Reproduction, Fertility, and Development $\mathbf{2 4}$ 59-67. (https://doi.org/10.1071/RD11907)

McPherson NO, Bell VG, Zander-Fox DL, Fullston T, Wu LL, Robker RL \& Lane M 2015 When two obese parents are worse than one! Impacts on embryo and fetal development. American Journal of Physiology: Endocrinology and Metabolism 309 E568-E581. (https://doi. org/10.1152/ajpendo.00230.2015)

Minge CE, Bennett BD, Norman RJ \& Robker RL 2008 Peroxisome proliferator-activated receptor- $\gamma$ agonist rosiglitazone reverses the adverse effects of diet-induced obesity on oocyte quality. Endocrinology 149 2646-2656. (https://doi.org/10.1210/en.2007-1570)

Mirabi P, Chaichi MJ, Esmaeilzadeh S, Jorsaraei SGA, Bijani A \& Ehsani M 2017 Does different BMI influence oocyte and embryo quality by inducing fatty acid in follicular fluid? Taiwanese Journal of Obstetrics and Gynecology 56 159-164. (https://doi.org/10.1016/j. tjog.2016.11.005)

Nichols J, Zevnik B, Anastassiadis K, Niwa H, Klewe-Nebenius D, Chambers I, Scholer H \& Smith A 1998 Formation of pluripotent stem cells in the mammalian embryo depends on the POU transcription factor Oct-4. Cell 95 379-391. (https://doi.org/10.1016/s00928674(00)81769-9)

Nishioka N, Inoue K, Adachi K, Kiyonari H, Ota M, Ralston A, Yabuta N, Hirahara S, Stephenson RO, Ogonuki N et al. 2009 The Hippo signaling pathway components Lats and Yap pattern Tead4 activity to distinguish mouse trophectoderm from inner cell mass. Developmental Cell $\mathbf{1 6}$ 398-410. (https://doi.org/10.1016/j.devcel.2009.02.003)

Niu Z, Lin N, Gu R, Sun Y \& Feng Y 2014 Associations Between insulin resistance, free fatty acids, and oocyte quality in polycystic ovary syndrome during in vitro fertilization. Journal of Clinical Endocrinology and Metabolism 99 E2269-E2276. (https://doi.org/10.1210/jc.20133942)

Niwa H, Toyooka Y, Shimosato D, Strumpf D, Takahashi K, Yagi R \& Rossant J 2005 Interaction between Oct3/4 and $\mathrm{Cdx} 2$ determines trophectoderm differentiation. Cell 123 917-929. (https://doi. org/10.1016/j.cell.2005.08.040)

O'Gorman A, Wallace M, Cottell E, Gibney MJ, McAuliffe FM, Wingfield M \& Brennan L 2013 Metabolic profiling of human follicular fluid identifies potential biomarkers of oocyte developmental competence. Reproduction 146 389-395. (https://doi.org/10.1530/REP-13-0184)

Ota M \& Sasaki H 2008 Mammalian tead proteins regulate cell proliferation and contact inhibition as transcriptional mediators of Hippo signaling. Development 135 4059-4069. (https://doi.org/10.1242/dev.027151)

Paeratakul S, Lovejoy JC, Ryan DH \& Bray GA 2002 The relation of gender, race and socioeconomic status to obesity and obesity comorbidities in a sample of US adults. International Journal of Obesity and Related Metabolic Disorders 26 1205-1210. (https://doi.org/10.1038/ sj.ijo.0802026)

Paim LMG \& FitzHarris G 2019 Tetraploidy causes chromosomal instability in acentriolar mouse embryos. Nature Communications 104834. (https://doi.org/10.1038/s41467-019-12772-8)

Paim LMG \& FitzHarris G 2020 The impact of embryo binucleation depends upon its origin. Reproduction $160 \mathrm{~V} 1-\mathrm{V} 4$. (https://doi.org/10.1530/REP20-0188)

Palomer X, Pizarro-Delgado J, Barroso E \& Vázquez-Carrera M 2018 Palmitic and oleic acid: the yin and yang of fatty acids in type 2 diabetes mellitus. Trends in Endocrinology and Metabolism 29 178-190. (https:// doi.org/10.1016/j.tem.2017.11.009)

Papaionnou VE \& Ebert KM 1995 Mouse half embryos: viability and allocation of cells in the blastocyst. Developmental Dynamics 203 393-398. (https://doi.org/10.1002/aja.1002030402)

Pfeffer PL 2018 Building principles for constructing a mammalian blastocyst embryo. Biology 7 41. (https://doi.org/10.3390/biology7030041) 
Piliszek A, Grabarek JB, Frankenberg SR \& Plusa B 2016 Cell fate in animal and human blastocysts and the determination of viability. Molecular Human Reproduction 22 681-690. (https://doi.org/10.1093/molehr/ gaw002)

Rodrigo R, Miranda-Merchak A, Valenzuela Grau R, Bachler JP \& Vergara L 2014 Modulation of ( $\mathrm{Na}, \mathrm{K})$-ATPase activity by membrane fatty acid composition: therapeutic implications in human hypertension. Clinical and Experimental Hypertension 36 17-26. (https://doi.org/10.3109/106 41963.2013.783048)

Sasaki H 2017 Roles and regulations of Hippo signaling during preimplantation mouse development. Development, Growth and Differentiation 59 12-20. (https://doi.org/10.1111/dgd.12335)

Schliwa M \& van Blerkom J 1981 Structural interaction of cytoskeletal components. Journal of Cell Biology 90 222-235. (https://doi. org/10.1083/jcb.90.1.222)

Schummers L, Hutcheon JA, Bodnar LM, Lieberman E \& Himes KP 2015 Risk of adverse pregnancy outcomes by prepregnancy body mass index: a population-based study to inform prepregnancy weight loss counselling. Obstetrics and Gynecology 125 133-143. (https://doi. org/10.1097/AOG.0000000000000591)

Staessen C \& Van Steirteghem A 1998 The genetic constitution of multinuclear blastomeres and their derivative daughter blastomeres. Human Reproduction 13 1625-1631. (https://doi.org/10.1093/ humrep/13.6.1625)

Stang J \& Huffman LG 2016 Position of the Academy of Nutrition and Dietetics: obesity, reproduction and pregnancy outcomes. Journal of the Academy of Nutrition and Dietetics 116 677-691. (https://doi. org/10.1016/j.jand.2016.01.008)

Strumpf D, Mao CA, Yamanaka Y, Ralston A, Chawengsaksophak K, Beck F \& Rossant J 2005 Cdx2 is required for correct cell fate specification and differentiation of trophectoderm in the mouse blastocyst. Development 132 2093-2102. (https://doi.org/10.1242/dev.01801)

Tarkowski AK 1959 Experiments on the development of isolated blastomeres of mouse eggs. Nature 184 1286-1287. (https://doi. org/10.1038/1841286a0)

Valckx SDM, Arias-Alvarez M, De Pauw I, Fievez V, Vlaeminck B, Fransen E, Bols PE \& Leroy JLMR 2014 Fatty acid composition of the follicular fluid of normal weight, overweight and obese women undergoing assisted reproductive treatment: a descriptive cross-sectional study. Reproductive Biology and Endocrinology 12 13. (https://doi.org/10.1186/1477-782712-13)

Violette MI, Madan P \& Watson AJW $2006 \mathrm{Na}^{+} / \mathrm{K}^{+}$-ATPase regulates tight junction formation and function during mouse preimplantation development. Developmental Biology 289 406-419. (https://doi. org/10.1016/j.ydbio.2005.11.004)
Wang H, Ding T, Brown N, Yamamoto Y, Prince LS, Reese J \& Paria BC 2008 Zonula occludens-1 (ZO-1) is involved in morula to blastocyst transformation in the mouse. Developmental Biology 318 112-125. (https://doi.org/10.1016/j.ydbio.2008.03.008)

Watson AJ \& Barcroft LC 2001 Regulation of blastocyst formation. Frontiers in Bioscience 6 D708-D730. (https://doi.org/10.2741/watson)

Wise LA, Rothman KJ, Mikkelson EM, Sorensen HT, Riis A \& Hatch EE 2010 An internet-based prospective study of body size and time-topregnancy. Human Reproduction 25 253-264. (https://doi.org/10.1093/ humrep/dep360)

World Health Organization 2000 Obesity: Preventing and Managing the Global Epidemic. Available at https://apps.who.int/iris/ handle/10665/42330 and accessed on 18 June 2020.

Wu LL, Russell DL, Norman RJ \& Robker RL 2012 Endoplasmic reticulum (ER) stress in cumulus-oocyte complexes impairs pentraxin-3 secretion, mitochondrial membrane potential $(\Delta \Psi \mathrm{m})$, and embryo development. Molecular Endocrinology 26 562-573. (https://doi.org/10.1210/ me.2011-1362)

Yousif MD, Calder MD, Du JT, Ruetz KN, Crocker K, Urquhart BL, Betts DH, Abu Rafea BA \& Watson AJ 2020 Oleic acid counters impaired blastocyst development induced by palmitic acid during mouse preimplantation development: understanding obesity related declines in fertility. Reproductive Sciences 27 2038-2051. (https://doi. org/10.1007/s43032-020-00223-5)

Yuan L, Mao Y, Luo W, Wu W, Xu H, Wang XL \& Shen YH 2017 Palmitic acid dysregulates the Hippo-YAP pathway and inhibits angiogenesis by inducing mitochondrial damage and activating the cytosolic DNA sensor cGAS-STING-IRF3 signaling mechanism. Journal of Biological Chemistry 292 15002-15015. (https://doi.org/10.1074/jbc.M117.804005)

Yuzefovych L, Wilson G \& Rachek L 2010 Different effects of oleate vs. palmitate on mitochondrial function, apoptosis, and insulin signaling in L6 skeletal muscle cells: role of oxidative stress. American Journal of Physiology: Endocrinology and Metabolism 299 E1096-E1105. (https:// doi.org/10.1152/ajpendo.00238.2010)

Zhang J, Yang Y \& Wu J 2010 Palmitate impairs cytokinesis associated with RhoA inhibition. Cell Research 20 492-494. (https://doi.org/10.1038/ cr.2010.33)

Received 3 September 2021

First decision 28 October 2021

Revised manuscript received 4 January 2022

Accepted 13 January 2022 